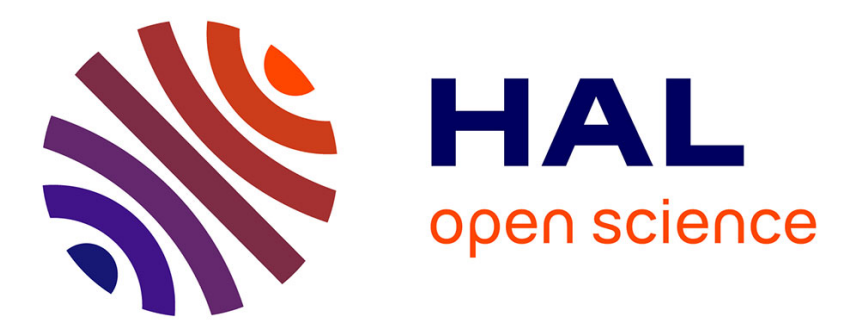

\title{
The electrostatic energy of a lattice of point charges
}

J. Olives

\section{To cite this version:}

J. Olives. The electrostatic energy of a lattice of point charges. Journal de Physique Lettres, 1985, 46

(24), pp.1143-1149. 10.1051/jphyslet:0198500460240114300 . jpa-00232949

\section{HAL Id: jpa-00232949 https://hal.science/jpa-00232949}

Submitted on 1 Jan 1985

HAL is a multi-disciplinary open access archive for the deposit and dissemination of scientific research documents, whether they are published or not. The documents may come from teaching and research institutions in France or abroad, or from public or private research centers.
L'archive ouverte pluridisciplinaire HAL, est destinée au dépôt et à la diffusion de documents scientifiques de niveau recherche, publiés ou non, émanant des établissements d'enseignement et de recherche français ou étrangers, des laboratoires publics ou privés. 


\title{
LE JOURNAL DE PHYSIQUE-LETTRES
}

J. Physique Lett. 46 (1985) L-1143 - L-1149

15 DÉCEMBRE 1985, PAGE L-1143

Classification

Physics Abstracts

$41.10 \mathrm{D}-61.50 \mathrm{~L}$

\section{The electrostatic energy of a lattice of point charges}

\section{J. Olives}

CRMC2, Campus de Luminy, 13288 Marseille Cedex 9, France

(Reçu le 13 juin 1985, accepté sous forme définitive le 21 octobre 1985)

\begin{abstract}
Résumé. - L'énergie électrostatique limite par maille $E$ d'un réseau ionique de charges ponctuelles est définie comme la valeur limite de l'énergie d'un cristal fini divisée par son nombre de mailles (lorsque ce nombre tend vers $+\infty$ ). La valeur explicite de $E$ est calculée, et reliée à l'énergie d'Ewald. Cette énergie $E$ dépend de la suite des cristaux finis croissants (plus précisément, de la suite des ensembles finis croissants d'ions). En d'autres termes, et brièvement : $E$ est une énergie volumique qui dépend de la surface.
\end{abstract}

\begin{abstract}
The limit electrostatic energy per cell $E$ of a ionic lattice of point charges is defined as the limit value of the energy of a finite crystal divided by its number of cells (when this number tends towards $+\infty$ ). The explicit value of $E$ is calculated and related to the Ewald energy. This energy $E$ depends on the sequence of the increasing finite crystals (more precisely, on the sequence of the increasing finite sets of ions). In other words, and briefly : $E$ is a volumic energy which depends on the surface.
\end{abstract}

\section{Introduction.}

The electrostatic energy of an ionic lattice, in which the ions are considered as point charges (with electrical neutrality of the cell), may be written as

$$
\begin{aligned}
E & =\sum_{\substack{\{\mathbf{n}+\mathbf{s}, \mathbf{p}+\mathbf{t}\} \\
\mathbf{n}+\mathbf{s} \neq \mathbf{p}+\mathbf{t}}} \frac{q_{\mathbf{s}} q_{\mathbf{t}}}{\|\mathbf{n}+\mathbf{s}-\mathbf{p}-\mathbf{t}\|} \\
& =\frac{1}{2} \sum_{\substack{\mathbf{n} \\
\mathbf{n}+\mathbf{s} \neq \mathbf{p}+\mathbf{t}}} \sum_{\substack{\mathbf{t} \\
\sum_{\mathbf{s}}}} \sum_{\mathbf{t}+\mathbf{s}-\mathbf{p}-\mathbf{t} \|} \frac{q_{\mathbf{s}} q_{\mathbf{t}}}{\| \mathbf{n}+\mathbf{s}-{ }^{\prime}}
\end{aligned}
$$


where $\mathbf{n}=n_{1} \mathbf{a}_{1}+n_{2} \mathbf{a}_{2}+n_{3} \mathbf{a}_{3}$ and $\mathbf{p}=p_{1} \mathbf{a}_{1}+p_{2} \mathbf{a}_{2}+p_{3} \mathbf{a}_{3}\left(\mathbf{a}_{1}, \mathbf{a}_{2}, \mathbf{a}_{3}\right.$ are cell vectors; $n_{1}, n_{2}, n_{3}, p_{1}, p_{2}, p_{3}$ are integers); $\mathbf{s}, \mathbf{t}$ are the positions of the ions of the origin cell $(\mathbf{n}=\mathbf{0}) ; q_{\mathbf{s}}$ is the charge of the ion s. Ewald [1] and Bertaut [2] considered that this energy may be written as

$$
E=\frac{1}{2} \sum_{\mathbf{p}} \sum_{\substack{\mathbf{m}+\mathbf{s} \neq \mathbf{t} \\ \mathbf{m}+\mathbf{t}}} \sum_{\mathbf{t}} \frac{q_{\mathbf{s}} q_{\mathbf{t}}}{\|\mathbf{m}+\mathbf{s}-\mathbf{t}\|}
$$

where $\mathbf{m}=\mathbf{n}-\mathbf{p}$ is supposed to run over all the lattice vectors; thus

$$
E=\frac{1}{2} N \sum_{\substack{\mathbf{m} \\ \mathbf{m}+\mathbf{s} \neq \mathbf{t}}} \sum_{\substack{\mathbf{t}\\}} \frac{q_{\mathbf{s}} q_{\mathbf{t}}}{\|\mathbf{m}+\mathbf{s}-\mathbf{t}\|}
$$

where $N$ is the number of cells, which leads to the electrostatic energy per cell :

$$
E_{0}=\frac{1}{2} \sum_{\substack{\mathbf{m} \\ \mathbf{m}+\mathbf{s} \neq \mathbf{t}}} \sum_{\mathbf{s}} \sum_{\mathbf{t}} \frac{q_{\mathbf{s}} q_{\mathrm{t}}}{\|\mathbf{m}+\mathbf{s}-\mathbf{t}\|}
$$

Ewald [1] and Bertaut [2] obtained, for this energy, the value

$$
E_{0}=E_{\mathrm{E}}
$$

with

$$
\begin{aligned}
& E_{\mathrm{E}}=E_{1}-E_{2}+E_{3} \\
& E_{1}=\frac{1}{2 \pi V} \sum_{\mathbf{h} \neq \mathbf{0}} \frac{|F(\mathbf{h}) \varphi(\mathbf{h})|^{2}}{\mathbf{h}^{2}} \\
& E_{2}=2 \pi \sum_{\mathrm{s}} q_{\mathrm{s}}^{2} \int_{0}^{+\infty} u p(u) \mathrm{d} u \\
& =2 \sum_{\mathbf{s}} q_{\mathrm{s}}^{2} \int_{0}^{+\infty}(\varphi(h))^{2} \mathrm{~d} h \\
& E_{3}=\frac{1}{2} \sum_{\substack{\mathbf{m} \\
\mathbf{m}+\mathbf{s} \neq \mathbf{t}}} \sum_{\substack{\mathbf{t} \\
\sum_{\mathbf{s}}}} \frac{q_{\mathrm{s}} q_{\mathrm{t}}}{\|\mathbf{m}+\mathbf{s}-\mathbf{t}\|} \int_{\|\mathbf{m}+\mathbf{s}-\mathbf{t}\|}^{+\infty} 4 \pi u(u-\|\mathbf{m}+\mathbf{s}-\mathbf{t}\|) p(u) \mathrm{d} u \\
& =\frac{1}{2} \sum_{\substack{\mathbf{m} \\
\mathbf{m}+\mathbf{s} \neq \mathbf{t}}} \sum_{\substack{\mathbf{t} \\
\mathbf{t}}} \frac{q_{\mathbf{s}} q_{\mathbf{t}}}{\|\mathbf{m}+\mathbf{s}-\mathbf{t}\|}\left(1-\frac{2}{\pi} \int_{0}^{+\infty}(\varphi(h))^{2} \frac{\sin (2 \pi h\|\mathbf{m}+\mathbf{s}-\mathbf{t}\|)}{h} \mathrm{~d} h\right)
\end{aligned}
$$

where $V$ is the volume of the cell $; \mathbf{h}$ is a vector of the reciprocal lattice; $F(\mathbf{h})=\sum_{\mathbf{s}} q_{\mathbf{s}} \mathrm{e}^{2 \pi i \mathrm{~h} . \mathbf{s}}$; $p$ and $\varphi$ are defined by

$$
\begin{aligned}
& p(\mathbf{u})=\int \sigma(\mathbf{x}) \sigma(\mathbf{x}+\mathbf{u}) \mathrm{d} \mathbf{x} \\
& \varphi(\mathbf{h})=\int \sigma(\mathbf{x}) \mathrm{e}^{2 \pi i \mathbf{h} . \mathbf{x}} \mathrm{d} \mathbf{x}
\end{aligned}
$$

( $p(\mathbf{u})$ depends only on $u=\|\mathbf{u}\|$ and $\varphi(\mathbf{h})$ on $h=\|\mathbf{h}\|)$, the function $\sigma$ being non-negative, 
of spherical symmetry and such that

$$
\int \sigma(\mathbf{x}) \mathrm{d} \mathbf{x}=1 .
$$

De Leeuw et al. [3] calculated the value of $E_{0}$, for a cubic lattice, and with the spherical mode of summation :

$$
E_{0}=\frac{1}{2} \lim _{N \rightarrow+\infty} \sum_{\|\mathbf{m}\| \leqslant N} \sum_{\substack{\mathbf{s}+\mathbf{s} \neq \mathbf{t} \\ \mathbf{t}}} \sum_{\mathbf{t}} \frac{q_{\mathbf{s}} q_{\mathbf{t}}}{\|\mathbf{m}+\mathbf{s}-\mathbf{t}\|}
$$

they found

$$
\begin{aligned}
E_{0} & =E_{1}-E_{2}+E_{3}+E_{4} \\
& =E_{\mathrm{E}}+E_{4}
\end{aligned}
$$

with the values of $E_{1}, E_{2}$ and $E_{3}$ obtained by Ewald and

$$
E_{4}=\frac{2 \pi \mathbf{M}^{2}}{3 a^{3}}
$$

where $a$ is the side length of the cubic cell and $\mathbf{M}=\sum_{\mathbf{s}} q_{\mathbf{s}} \mathbf{s}$ (dipole moment of the cell). Smith [4] extended the preceding result to other modes of summation.

In this paper, we present two results : in section 2, the calculation of $E_{0}$, and its relation to the Ewald energy $E_{\mathrm{E}}$; in section 3, the calculation of the limit electrostatic energy per cell $E$ (defined in section 3), and its relation to $E_{0}$ (and then to $E_{\mathrm{E}}$ ). These results hold for an arbitrary lattice of point charges - in which the cell is electrically neutral, but its dipole moment may be different from $\mathbf{0}$ - and for arbitrary modes of summation.

\section{Calculation of $E_{0}$.}

The mathematical proof corresponding to this section has been given in another paper [5]. We have observed that the result (3)-(4) of Ewald and Bertaut may be considered as an incorrect application of Poisson's formula to the function $f$

$$
\begin{aligned}
& f(\mathbf{h})=\frac{|F(\mathbf{h}) \varphi(\mathbf{h})|^{2}}{\mathbf{h}^{2}}, \quad \mathbf{h} \neq \mathbf{0} \\
& f(\mathbf{0})=0,
\end{aligned}
$$

since $f$ is not continuous at $\mathbf{h}=\mathbf{0}$ (if the dipole moment of the cell $\mathbf{M}=\sum_{\mathbf{s}} q_{\mathbf{s}} \mathbf{s}$ is different from $\mathbf{0}$ ). We have then replaced the function $f$ by a sequence of continuous functions $f_{N}-$ to which Poisson's formula may be applied - and we have taken the limit of Poisson's formula as $N \rightarrow+\infty$.

We obtain the following result, if $\mathbf{M} \neq \mathbf{0}$ :

Let $\left(B_{k}\right)$ be any sequence of finite subsets of $\mathbb{Z}^{3}$ such that

(i) $\mathrm{B}_{k} \subset \mathrm{B}_{k+1}$, for all $k$;

(ii) $\bigcup_{k} \mathrm{~B}_{k}=\mathbb{Z}^{3}$;

(iii) $\sum_{m \in B_{k} \backslash\{0\}} \frac{1-3 \cos ^{2} \theta_{\mathbf{m}}}{\|\mathbf{m}\|^{3}}$ has a finite limit as $k \rightarrow+\infty$; notations : $m=\left(m_{1}, m_{2}, m_{3}\right) \in \mathbb{Z}^{3}$; and $\theta_{\mathbf{m}}$ is the angle between $\mathbf{M}$ and $\mathbf{m}$. 
Then

$$
E_{0}=\frac{1}{2} \lim _{k \rightarrow+\infty} \sum_{\substack{\mathbf{m} \in \mathbf{B}_{k} \\ \mathbf{m}+\mathbf{s} \neq \mathbf{t}}} \sum_{\mathbf{s}} \sum_{\mathbf{t}} \frac{q_{\mathbf{s}} q_{\mathbf{t}}}{\|\mathbf{m}+\mathbf{s}-\mathbf{t}\|}
$$

exists and is equal to

$$
\begin{aligned}
E_{0} & =E_{1}-E_{2}+E_{3}+E_{4}+E_{5}-E_{5}^{0} \\
& =E_{\mathrm{E}}+E_{4}+E_{5}-E_{5}^{0}
\end{aligned}
$$

where $E_{1}, E_{2}$ and $E_{3}$ are given by (4) and

$$
\begin{aligned}
& E_{4}=\frac{2 \pi \mathbf{M}^{2}}{3 V} \\
& E_{5}=\frac{\mathbf{M}^{2}}{2} \lim _{k \rightarrow+\infty} \sum_{m \in \mathbf{B}_{k} \backslash\{0\}} \frac{1-3 \cos ^{2} \theta_{\mathbf{m}}}{\|\mathbf{m}\|^{3}} \\
& E_{5}^{0}=\frac{\mathbf{M}^{2}}{2} \lim _{N \rightarrow+\infty} \sum_{0<\|\mathbf{m}\| \leqslant N} \frac{1-3 \cos ^{2} \theta_{\mathbf{m}}}{\|\mathbf{m}\|^{3}} .
\end{aligned}
$$

If $\mathbf{M}=\mathbf{0}$, the sum

$$
E_{0}=\frac{1}{2} \sum_{\substack{\mathbf{m} \\ \mathbf{m}+\mathbf{s} \neq \mathbf{t}}} \sum_{\mathbf{s}} \sum_{\substack{\mathbf{t} \\ \mathbf{m}}} \frac{q_{\mathbf{t}}}{\|\mathbf{m}-\mathbf{t}\|}
$$

is absolutely convergent and

$$
\begin{aligned}
E_{0} & =E_{1}-E_{2}+E_{3} \\
& =E_{\mathrm{E}} .
\end{aligned}
$$

The term $E_{4}$ generalizes the expression (7) found by de Leeuw et al. [3] for cubic crystals (Felderhof [6] obtained this term from a virtual uniform polarization $\mathbf{M} / V)$. The terms $E_{5}$ and $E_{5}^{0}$ represent two sums of the same family of numbers $\left(\frac{\mathbf{M}^{2}\left(1-3 \cos ^{2} \theta_{\mathbf{m}}\right)}{2\|\mathbf{m}\|^{3}}\right)_{\mathbf{m} \neq \mathbf{0}}$, with the mode of summation $\left(\mathrm{B}_{k}\right)$ for $E_{5}$ (as for $E_{0}$ ), and the spherical mode of summation for $E_{5}^{0}$. The contribution $E_{5}-E_{5}^{0}$ is generally different from 0 , if $\mathbf{M} \neq \mathbf{0}$ and $\left(\mathrm{B}_{k}\right)$ is not the spherical mode of summation.

\section{The limit electrostatic energy per cell $E$.}

We may note that the considerations of the introduction leading to (2) are contradictory : the crystal is supposed to be at the same time infinite ( $m$ runs over the whole lattice) and finite ( $N$ cells). The limit electrostatic energy per cell $E$ may be correctly defined as the limit of the energy of a finite crystal (formed by a finite number of cells) divided by its number of cells (when this number tends towards $+\infty)$ :

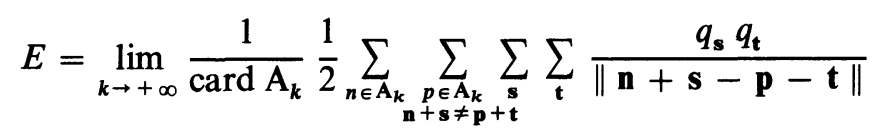

where $\left(A_{k}\right)$ is an increasing sequence of finite subsets of $\mathbb{Z}^{3}$ such that $\bigcup A_{k}=\mathbb{Z}^{3}$ (each $A_{k}$ represents the shape of a finite crystal.). 
3. 1 ResUlt. - Let $\left(A_{k}\right)$ be any sequence of finite subsets of $\mathbb{Z}^{3}$ such that

1) $\mathrm{A}_{k} \subset \mathrm{A}_{k+1}$, for all $k$;

2) $\bigcup_{k} A_{k}=\mathbb{Z}^{3}$;

3) if $\mathbf{M} \neq \mathbf{0}$, we suppose that $\sum_{m \in \mathbf{B}_{k} \backslash\{0\}} \frac{1-3 \cos ^{2} \theta_{\mathbf{m}}}{\|\mathbf{m}\|^{3}}$ has a finite limit as $k \rightarrow+\infty$; notations : $\mathbf{B}_{k}=\left\{n-p \mid n\right.$ and $\left.p \in \mathbf{A}_{k}\right\} ; \theta_{\mathbf{m}}=$ angle between $\mathbf{M}$ and $\mathbf{m}$;

4) there exists $(f(k)), a>0, b \geqslant 0, \alpha>0$, such that

4a) $a . d(k) \leqslant f(k)$, for all $k$; notation : $d(k)=\sup _{n \in \mathbf{A}_{\boldsymbol{k}}}\|\mathbf{n}\|$;

4b) $1-c\left(\mathbf{A}_{k}, m\right) \leqslant b\left(\frac{\|\mathbf{m}\|}{f(k)}\right)^{\alpha}$, for all $k$ and $m \in \mathbf{B}_{k}$; notation $c\left(\mathbf{A}_{k}, m\right)=\frac{\operatorname{card}\left(\mathbf{A}_{k} \cap m+\mathbf{A}_{k}\right)}{\operatorname{card} \mathbf{A}_{k}}$;

4c) if $\mathbf{M} \neq \mathbf{0}$, we suppose that, for almost every $x \in \mathbb{R}^{3}, c\left(\mathrm{~A}_{k}, m_{k}\right)$ has a finite limit as $k \rightarrow+\infty$, which is denoted by $c(x) . m_{k}$ is defined by $x \in \frac{1}{f(k)}\left(m_{k}+\mathrm{C}\right) ; \mathrm{C}=\left[0,1\left[^{3}\right.\right.$;

4d) if $\mathbf{M} \neq \mathbf{0}$, we suppose that, for almost every $x \in \mathbb{R}^{3}, \varphi_{\frac{1}{f^{(k)}}\left(\mathbf{B}_{\boldsymbol{k}}+\mathrm{C}\right)}(x)$ has a limit as $k \rightarrow+\infty$, which is denoted by $\varphi_{\mathrm{B}}(x) . \varphi_{\mathrm{A}}$ is a characteristic function of a subset $A$ of $\mathbb{R}^{3}$.

Then the limit electrostatic energy per cell $E$ defined by (13) exists and is equal to

$$
E=E_{0}-E_{6}
$$

where

$$
\begin{gathered}
E_{0}=\lim _{k \rightarrow+\infty} \frac{1}{2} \sum_{\substack{m \in \mathbf{B}_{\mathbf{k}} \\
\mathbf{m}+\mathbf{s} \neq \mathbf{t}}} \sum_{\mathbf{s}} \sum_{\mathbf{t}} \frac{q_{\mathrm{s}} q_{\mathbf{t}}}{\|\mathbf{m}+\mathbf{s}-\mathbf{t}\|} \\
E_{6}=\frac{\mathbf{M}^{2}}{2 V} \int_{x \in \mathbf{B}}(1-c(x)) \frac{1-3 \cos ^{2} \theta_{\mathbf{x}}}{\|\mathbf{x}\|^{3}} \mathrm{dx}
\end{gathered}
$$

if $\mathbf{M} \neq \mathbf{0}\left(\theta_{\mathbf{x}}=\right.$ angle between $\mathbf{M}$ and $\left.\mathbf{x}\right)$; and, if $\mathbf{M}=\mathbf{0}$

where

$$
E=E_{0}
$$

$$
E_{0}=\frac{1}{2} \sum_{\substack{\mathbf{m} \\ \mathbf{m}+\mathbf{s} \neq \mathbf{t}}} \sum_{\substack{\mathbf{t}\\}} \frac{q_{\mathbf{s}} q_{\mathbf{t}}}{\|\mathbf{m}+\mathbf{s}-\mathbf{t}\|}
$$

(the sum on $\mathbf{m}$ is here absolutely convergent). In all cases, the value of $E_{0}$ is given in section 2.

\subsection{EXAMPLES. - If}

$$
\mathbf{A}_{k}=\{n \mid\|\mathbf{n}\| \leqslant k\}
$$

we have

$$
\begin{array}{lrl}
c\left(\mathrm{~A}_{k}, m\right) \approx 1-\frac{3\|\mathbf{m}\|}{4 k}+\frac{\|\mathbf{m}\|^{3}}{16 k^{3}} & \text { (for } k \text { and } 2 k-\|\mathbf{m}\| \gg 1) & \text { if }\|\mathbf{m}\| \leqslant 2 k, \\
c\left(\mathbf{A}_{k}, m\right)=0 & \text { if }\|\mathbf{m}\|>2 k .
\end{array}
$$

All the above conditions 1) to 4) are satisfied : condition 3) has been proved in [5], and condition 4) is satisfied with $f(k)=d(k)=k$ and $\alpha=1$. Since $c(x)$ depends only on $\|\mathbf{x}\|$, the integral $E_{6}$ is 
equal to 0 (with $M$ as polar axis, the integral on $\theta$ is equal to 0 ), so that

with

$$
E=E_{0}
$$

$$
E_{0}=\lim _{k \rightarrow+\infty} \frac{1}{2} \sum_{\substack{\|\mathbf{m}\| \leqslant k \\ \mathbf{m}+\mathbf{s} \neq \mathbf{s}}} \sum_{\substack{\mathbf{s} \\ \mathbf{t}}} \frac{q_{\mathbf{s}} q_{\mathbf{t}}}{\|\mathbf{m}+\mathbf{s}-\mathbf{t}\|}
$$

if $\mathbf{M} \neq \mathbf{0}$, and

if $\mathbf{M}=\mathbf{0}$.

$$
E_{0}=\frac{1}{2} \sum_{\substack{\mathbf{m} \\ \mathbf{m}+\mathbf{s} \neq \mathbf{t}}} \sum_{\mathbf{s}} \sum_{\substack{\mathbf{t} \\ \| \mathbf{s}}} \frac{q_{\mathbf{t}}}{\|\mathbf{m}+\mathbf{s}-\mathbf{t}\|}
$$

If

$$
\mathrm{A}_{k}=\{n|| n \mid \leqslant k\}
$$

(we note $|n|=\sup \left\{\left|n_{1}\right|,\left|n_{2}\right|,\left|n_{3}\right|\right\}$ ), we have

$$
\begin{array}{ll}
c\left(\mathrm{~A}_{k}, m\right)=\left(1-\frac{\left|m_{1}\right|}{2 k+1}\right)\left(1-\frac{\left|m_{2}\right|}{2 k+1}\right)\left(1-\frac{\left|m_{3}\right|}{2 k+1}\right) & \text { if }|m| \leqslant 2 k \\
c\left(\mathrm{~A}_{k}, m\right)=0 & \text { if }|m|>2 k,
\end{array}
$$

and the above condition 4) is satisfied $(f(k)=k$ and $\alpha=1)$. If condition 3) is also satisfied, we may then write

where

$$
E=E_{0}-E_{6}
$$

$$
\begin{aligned}
& E_{0}=\lim _{k \rightarrow+\infty} \frac{1}{2} \sum_{\substack{|m| \leqslant k \\
\mathbf{m}+\mathbf{s} \neq \mathbf{t}}} \sum_{\substack{\mathbf{t} \\
\mathbf{m}}} \frac{q_{\mathbf{s}} q_{\mathbf{t}}}{\|\mathbf{m}+\mathbf{s}-\mathbf{t}\|} \\
& E_{6}=\frac{\mathbf{M}^{2}}{2 V} \int_{|x| \leqslant 1}\left[1-\left(1-\left|x_{1}\right|\right)\left(1-\left|x_{2}\right|\right)\left(1-\left|x_{3}\right|\right)\right] \frac{1-3 \cos ^{2} \theta_{x}}{\|\mathbf{x}\|^{3}} \mathrm{~d} \mathbf{x}
\end{aligned}
$$

if $\mathbf{M} \neq \mathbf{0}$, or

$$
E=E_{0}
$$

where

$$
E_{0}=\frac{1}{2} \sum_{\substack{\mathbf{m} \\ \mathbf{m}+\mathbf{s} \neq \mathbf{t}}} \sum_{\substack{\mathbf{t} \\ \mathbf{m}}} \frac{q_{\mathbf{s}} q_{\mathbf{t}}}{\|\mathbf{m}+\mathbf{s}-\mathbf{t}\|}
$$

if $\mathbf{M}=\mathbf{0}$.

\section{Numerical example.}

As a numerical example, we have computed the different terms in a simple case : orthogonal cell vectors $\mathbf{a}_{1}, \mathbf{a}_{2}, \mathbf{a}_{3}$ with $\left\|\mathbf{a}_{1}\right\|=\left\|\mathbf{a}_{2}\right\|=1,\left\|\mathbf{a}_{3}\right\|=2, q_{\mathbf{s}}=+1$ at $\mathbf{s}=\mathbf{0}, q_{\mathbf{s}}=-1$ at $\mathbf{s}=\frac{1}{2}\left(\mathbf{a}_{1}+\mathbf{a}_{2}+\mathbf{a}_{3}\right)$.

Calculation of $E_{0}$ : we have found : $E_{1}-E_{2}+E_{3}=E_{\mathrm{E}}=-0.74$ (calculation time $\sim 5 \mathrm{~s}$ ), $E_{4}=1.57, E_{5}=1.33$ with the " $|n|$ mode of summation" $\left(\mathrm{B}_{k}=\left\{n|| n \mid=\sup \left(\left|n_{1}\right|,\left|n_{2}\right|\right.\right.\right.$, $\left.\left.\left|n_{3}\right|\right) \leqslant k\right\}$ ), $E_{5}^{0}=1.82$ (calculation time $\sim 20 \mathrm{~s}$ ); thus : $E_{1}-E_{2}+E_{3}+E_{4}=0.83$ (calculation time $\sim 5 \mathrm{~s}$ ), $E_{1}-E_{2}+E_{3}+E_{4}+E_{5}-E_{5}^{0}=0.34$ (calculation time $\sim 25 \mathrm{~s}$ ). These 
last two values have been also obtained by the direct calculation (8) of $E_{0}$, with, respectively, the spherical mode of summation and the $|n|$ mode of summation (with calculation times of $\sim 15 \mathrm{~s}$ ). All the calculations were made on a 16 bit minicomputer (PDP 11/45 of DEC).

Calculation of $E$ : With the spherical shape (16) and according to (17) and (18), we obtain $E=0.83$ (from the above value of $E_{0}$ ). With the $|n|$ shape (20) and according to (21) and (22), we obtain : $E_{0}=0.34$ (see above), $E_{6}=-0.17$ (calculation time $40 \mathrm{~min}$ ), so that $E=0.51$. These two values $E=0.83$ (spherical shape) and $E=0.51(|n|$ shape) may be directly calculated with the help of (13), but the convergence is extremely slow. For the spherical shape, we have obtained, $E=0.66$ at $k=8$ (calculation time $5 \mathrm{~h}$ ) and $E=0.69$ at $k=10$ (calculation time $15 \mathrm{~h}$ ); and, for the $|n|$ shape, $E=0.43$ at $k=8$ (calculation time $20 \mathrm{~h}$ ) and $E=0.44$ at $k=10$ (calculation time $62 \mathrm{~h}$ ). These results show that the expression (14)-(15) of $E$ is much more interesting for calculation than the definition (13).

\section{Conclusion.}

The results presented in sections 2 and 3 show that - under the conditions 1) to 4) of section 3.1 - the limit electrostatic energy per cell $E$ (defined by (13)) exists and is related to the Ewald energy $E_{\mathrm{E}}$ by

$$
\begin{aligned}
& E=E_{\mathbf{E}} \text { if } \mathbf{M} \text { (dipôle moment of the cell) }=\mathbf{0} \\
& E=E_{\mathrm{E}}+E_{4}+E_{5}-E_{5}^{0}-E_{6} \text { if } \mathbf{M} \neq \mathbf{0},
\end{aligned}
$$

where the terms $E_{4}, E_{5}$ and $E_{5}^{0}$ are given by (10) and $E_{6}$ by (14).

The finite crystals - used to define $E$ - are formed by the sets of ions

$$
\mathrm{S}_{k}=\left\{\mathbf{n}+\mathbf{s} / n \in \mathrm{A}_{\boldsymbol{k}}, \mathbf{s} \in \text { cell }\right\}
$$

( $\mathbf{n}$ are the lattice vectors which give the positions of the cells; $\mathbf{s}$ are the vectors which give the positions of the ions of the cell $\mathbf{n}=\mathbf{0}$ ).

For a given crystal, each $S_{k}$ depends on $A_{k}$ and on the cell which has been chosen. The dependence of all the terms $E_{4}, E_{5}, E_{5}^{0}$ and $E_{6}$ on $\mathbf{M}$ (which depends on the cell), clearly shows the dependence of $E$ on the choice of the cell (for a given crystal). The dependence of $E$ on the sequence $\left(\mathrm{A}_{k}\right)$ appears in the terms $E_{5}$ and $E_{6}\left(\left(\mathrm{~B}_{k}\right), \mathrm{B}\right.$ and $c(x)$ depend on $\left.\left(\mathrm{A}_{k}\right)\right)$.

\section{References}

[1] Ewald, P. P., Ann. Physik 64 (1921) 253.

[2] Bertaut, F., J. Phys. Rad. 13 (1952) 499.

[3] De Leeuw, S. W., Perram, J. W. and Smith, E. R., Proc. R. Soc. London A 373(1980) 27.

[4] Smith, E. R., Proc. R. Soc. London A 375 (1981) 475.

[5] Olives, J., 1985, submitted to J. Phys. A.

[6] Felderhof, B. U., Physica A 101 (1980) 275. 